Article

\title{
Entamoeba histolytica: Proteomics Bioinformatics Reveal Predictive Functions and Protein-Protein Interactions of Differentially Abundant Membrane and Cytosolic Proteins
}

\author{
Norhidayah Azmi and Nurulhasanah Othman *(D) \\ Institute for Research in Molecular Medicine (INFORMM), Universiti Sains Malaysia, \\ Pulau Pinang 11800, Malaysia; hidayah84@student.usm.my \\ * Correspondence: nurulhasanah@usm.my
}

check for

updates

Citation: Azmi, N.; Othman, N. Entamoeba histolytica: Proteomics Bioinformatics Reveal Predictive Functions and Protein-Protein Interactions of Differentially Abundant Membrane and Cytosolic Proteins. Membranes 2021, 11, 376. https://doi.org/10.3390/

membranes 11060376

Academic Editor: Igor Zhukov

Received: 30 March 2021

Accepted: 18 May 2021

Published: 21 May 2021

Publisher's Note: MDPI stays neutral with regard to jurisdictional claims in published maps and institutional affiliations.

Copyright: (c) 2021 by the authors. Licensee MDPI, Basel, Switzerland. This article is an open access article distributed under the terms and conditions of the Creative Commons Attribution (CC BY) license (https:/ / creativecommons.org/licenses/by/ $4.0 /)$.

\begin{abstract}
Amoebiasis is caused by Entamoeba histolytica and ranked second for parasitic diseases causing death after malaria. E. histolytica membrane and cytosolic proteins play important roles in the pathogenesis. Our previous study had shown several cytosolic proteins were found in the membrane fraction. Therefore, this study aimed to quantify the differential abundance of membrane and cytosolic proteins in membrane versus cytosolic fractions and analyze their predicted functions and interaction. Previous LC-ESI-MS/MS data were analyzed by PERSEUS software for the differentially abundant proteins, then they were classified into their functional annotations and the protein networks were summarized using PantherDB and STRiNG, respectively. The results showed $24(44.4 \%)$ out of the 54 proteins that increased in abundance were membrane proteins and 30 were cytosolic proteins. Meanwhile, 45 cytosolic proteins were found to decrease in abundance. Functional analysis showed differential abundance proteins involved in the molecular function, biological process, and cellular component with $18.88 \%, 33.04 \%$ and, $48.07 \%$, respectively. The STRiNG server predicted that the decreased abundance proteins had more protein-protein network interactions compared to increased abundance proteins. Overall, this study has confirmed the presence of the differentially abundant membrane and cytosolic proteins and provided the predictive functions and interactions between them.
\end{abstract}

Keywords: Entamoeba histolytica; membrane protein; cytosolic protein; differential protein abundance; functional annotation; protein-protein interaction

\section{Introduction}

Amoebiasis or amoebic dysentery is a protozoan disease caused by Entamoeba histolytica, which is mainly found in the human colon. It may exist as a non-pathogenic commensal or penetrate the intestinal mucosa and metastasize to cause an extraintestinal infection like an amoebic liver abscess (ALA) [1]. The parasite has a simple two-stage life cycle including trophozoite and cyst stages. Infection of a human by E. histolytica begins by ingestion of the cyst, which is protected from the environment by a highly resistant chitin-containing cell wall [2]. Trophozoites penetrate the intestinal mucus layer that develops colitis in the colon during the disease-causing process. Trophozoite invasion includes the destruction of epithelial cells, lymphocytes, and polymorphonuclear cells [2].

Many studies have been performed on the trophozoites form because it is easy to cultivate. During the infection, surface molecules of trophozoites connect the host to the parasite, which is crucial in tissue invasion, colitis induction, and the development of liver abscess [3]. However, little is understood about the E. histolytica membrane molecules. Membrane proteins can be found on the cell surface, reticulum endoplasm (ER), Golgi apparatus, endomembrane, mitochondria and nucleus. In E. histolytica, host-parasite interactions occur on the membrane surface, which is the outer layer of the trophozoites, and the adherence process is initiated by Gal/GalNAc lectin membrane protein, which is 
the first step in developing the disease. This adherence is important in mediating killing and/or phagocytosis and exposing the surface molecules to the host's immune system [2].

Membrane proteins possess transmembrane (TM) domains, which differentiate them from cytosolic proteins [4]. They are responsible for cellular adhesion and recognition, molecular receptors, substrate transportation through membranes, signal transduction, protein secretion, enzymatic activity [5] and/or signal peptides, which assists protein positioning in cellular organelles or the cell membrane [6]. To date, only around 20 proteins or protein families of E. histolytica membrane have been identified [3]. Therefore, it is important to further identify E. histolytica membrane proteins since a majority of the protein functions are unexplored.

Critical steps for protein analysis in a biological context are extraction and isolation of proteins from chemical and physical interactions with other biomolecules from specific cells [7]. Hence, our previous extensive study (Ujang et al., (2018) [7]) performed three different extraction methods to fractionate membrane and cytosolic proteins of E. histolytica trophozoites. The extractions were performed to isolate the total membrane and not for an organelle specific-membrane fraction. The membrane and cytosolic fractions were analyzed by LC-ESI-MS/MS for protein identification to indicate the presence or absence of the membrane and cytosolic proteins in the fractions. The result showed a commercial kit, ProteoPrep (Sigma-Aldrich, Darmstadt, Germany) was the best method for the membrane protein extraction in terms of its sensitivity and specificity by analyzing membrane and cytosolic fractions. Mixed membrane and cytosolic proteins accounted for 267, 127 and 130 identified proteins in both fractions [7]. The cytosolic proteins were found to be present in the membrane fraction from the three extraction methods. This phenomenon has raised our curiosity as to whether the cytosolic proteins were really located and worked hand in hand with the membrane proteins or this phenomenon occurred because of the technique used during the membrane extraction step.

Using the previous MS data of label-free quantification [7], we aimed to analyze the differential abundance proteins in membrane versus cytosolic fractions of E. histolytica. The differentially abundant proteins were analyzed using bioinformatics platforms to further understand the predictive biological functions and interactions among the proteins. In this study, the TM and signal peptide domains of identified proteins were obtained from AmoebaDB database. The database uses a specific algorithm to predict the presence of these domains in the protein. We hypothesized that the membrane proteins were increased while cytosolic proteins were decreased in abundance by comparing the membrane versus cytosolic fractions. If there were increased abundance of membrane and cytosolic proteins in the membrane fraction, we postulated there were protein-protein interactions between these two proteins. This would therefore strengthen the notion that the cytosolic proteins localized to the membrane part and functioned together with membrane proteins in E. histolytica.

\section{Materials and Methods}

\subsection{PERSEUS Software Platform Analysis}

MS data of LFQ from three different extraction methods by Ujang et al., (2018) [7] were retrieved from ProteomeXchange with identifier no. PXD010171 for differential protein abundant analysis in membrane versus cytosolic fractions using PERSEUS MaxQuant software (MaxPlanck Institute of Biochemistry, Martinsried, Germany). The LFQ intensities were based on 267, 127 and 130 identified proteins in both fractions [7]. In this study, the LFQ intensities of the membrane fraction was the numerator and the cytosolic fraction was the denominator. Therefore, the differential abundance proteins presented in the result were on the proteins of the membrane fraction. The PERSEUS integrates a multitude of algorithms enabling complete analysis of MS data starting from raw LC-MS runs. Firstly, Perseus.exe was downloaded from the website www.maxquant.net/perseus / (accessed on: 06.05.2020). The Generic Matrix Upload icon was clicked, and the icon box was selected. A new window popped out. The data frame was composed by selecting data features 
carefully inside the right box. The LFQ intensities of membrane and cytosolic proteins of three extraction methods were uploaded for quantification and statistical analyses along with other parameters, i.e., accession no., description of protein, score, number of peptides and coverage. Log in transformation parameter and base parameters were selected to transform the data in order to facilitate the protein abundance fold change calculation in the analysis. For data analysis, there were four different statistical tests offered by Perseus; one sample, two samples, multiple samples, and two-way ANOVA. This study used t-test for two group analysis. The q-value and False Discovery Rate (FDR) calculated were set-up to show a cut-off curve indicating which proteins were significant. The S0 (fold-change) and FDR were set to 0 and $\mathrm{q}<0.05$, respectively. Then, a volcano plot was visualized by setting the $x$ and $y$-axis with $\log _{2}$ fold changes and $-\log _{10} p$-values, respectively. This was done to visualize the potential or significant proteins located in the right and left of the quadrant's plot. Finally, the statistical results were saved by exporting to a file or in .txt and it could be opened either in Excel or Perseus software. The proteins that showed differences in the fold-change $(\mathrm{Fc})>2$ and $\mathrm{q}<0.01$ were considered significant.

\subsection{Determination of Transmembrane and Signal Peptide Domains}

The accession no. of differentially abundant proteins was searched against the AmoebaDB database release 47 (accessed on 10 June 2020). Then, information on the presence of transmembrane and signal peptide domains were obtained from the protein properties and features section.

\subsection{Gene Ontology}

Classification of the differential abundant proteins into their molecular function, biological process, and cellular component was performed by the Protein ANalysis THrough Evolutionary Relationship (PANTHER) DB version 15.0 system at www.pantherdb.org (accessed on 17 June 2020). The Gene List Analysis page was browsed, the accession number of the increased or decreased abundant proteins with fold -change $\left(F_{c}\right)>2$ and $q<0.05$ were typed in the box and the ID list was ticked. Then, E. histolytica was selected as the ID and functional classification was viewed in the gene list. The submit button was clicked to generate pie charts. The result was based on the analysis that was selected, i.e., Functional Classification Viewed in the Gene List. It displayed a gene list indicating mapped and unmapped genes. The system brought up the family list page if PANTHER Generic Mapping file or ID's from Reference Proteome Genome file was selected as an input file. A multi-coloured pie chart icon was clicked to display the functional analysis results of all the proteins involved in molecular function, biological process, cellular components, and protein classes and their related pathways. Category name, and series of numbers appeared when the mouse was moved over the pie chart. The numbers represented statistics of the selected category. The legend link on the right side of the pie chart also could be used to retrieve a gene list of the category.

\subsection{Protein-Protein Interaction Network Prediction}

The www.string-db.org (version 11, accessed on 20 June 2020) webpage was navigated to analyze protein-protein interaction networks. STRiNG provides a database of known and predicted protein-protein interactions including indirect (functional) and direct (physical) associations arising from computational prediction, knowledge transferable and primary databases, such as participating in the same biological process or protein class (gene ontology). STRiNG (Search Tool for Retrieval of Interacting Genes/Proteins) can be searched by single or multiple protein names, or by amino acid sequences in any available formats. In this study, a total of 99 accession numbers derived from 54 and 45 increased and decreased abundant proteins was inserted as protein identifier. The multiple proteins input was selected. Then, the organism of interest (E. histolytica) was specified before clicking the SEARCH button. Then, the network was displayed to show the predicted network of association for a group of proteins. Proteins were represented by the nodes, and edges 
showed predicted functional associations. By clicking on a node gave several details of the protein and the edge displayed a detailed evidence breakdown. The line or edge could be seen in seven different colors, which represented different evidence used in protein relation prediction. The protein window provided information about the protein and other links related to the protein.

\section{Results}

\subsection{Differential Abundant Proteins}

By combining the results from mass spectrometry analysis of three extraction methods by Ujang et al., 2018 [7], a total of 99 differentially abundant proteins with 54 increased and 45 decreased abundance in membrane versus cytosolic fractions fulfilled the chosen criteria of protein fold-change $\left(\mathrm{Fc}_{\mathrm{c}}\right)>2$ fold and $\mathrm{q}<0.05$ (Table 1$)$. Furthermore, 24 of the increased abundant proteins were predicted as membrane proteins (Table 2) and there was no membrane protein from decreased abundance proteins (Table 3).

Table 1. Increased and decreased abundance proteins in the membrane versus cytosolic fractions $(\mathrm{q}<0.05)$ by three different methods.

\begin{tabular}{ccc}
\hline \multirow{2}{*}{ Extraction Method } & Increased & Decreased \\
\cline { 2 - 3 } & Fc $>$ 2-Fold; $\mathbf{q}<\mathbf{0 . 0 5}$ & Fc $>$ 2-Fold; $\mathbf{q}<\mathbf{0 . 0 5}$ \\
\hline Conventional Method & 19 & 23 \\
ProteoExtract Kit & 4 & 2 \\
ProteoPrep Kit & 31 & 20 \\
TotalL & 54 & 45 \\
\hline
\end{tabular}

Table 2. Increased abundant membrane and cytosolic proteins in the membrane versus cytosolic fractions $(q<0.05)$.

\begin{tabular}{|c|c|c|c|}
\hline TM and/or Signal Peptide & Fold Change & Mapped ID & Gene Name \\
\hline- & 2.0141 & EHI_078310 & Uncharacterized protein \\
\hline- & 2.3102 & EHI_129750 & Rho family GTPase \\
\hline- & 2.0333 & EHI_198580 & Uncharacterized protein \\
\hline Yes & 2.4443 & EHI_165070 & Estradiol 17-beta-dehydrogenase putative \\
\hline- & 2.6115 & EHI_023360 & Alpha-amylase family protein \\
\hline- & 3.6739 & EHI_065790 & Rab family GTPase (Fragment) \\
\hline- & 2.3510 & EHI_152280 & Serine palmitoyltransferase putative \\
\hline Yes & 2.6371 & EHI_199590 & $70 \mathrm{kDa}$ heat shock protein putative \\
\hline- & 2.2277 & EHI_120360 & Grainin putative \\
\hline- & 2.1083 & EHI_006980 & Gal galnac lectin subunit igl1 \\
\hline- & 2.5152 & EHI_102170 & Elongation factor 1-alpha \\
\hline Yes & 2.0661 & EHI_024350 & Uncharacterized protein \\
\hline- & 2.0284 & EHI_107290 & Actin \\
\hline- & 2.6989 & EHI_047800 & Uncharacterized protein \\
\hline Yes & 2.5246 & EHI_076870 & 3-oxo 5 alpha-steroid 4-dehydrogenase domain-containing \\
\hline Yes & 2.9713 & EHI_054830 & Calcium-transporting ATPase \\
\hline- & 4.4102 & EHI_050520 & Uncharacterized protein \\
\hline- & 2.8741 & EHI_169280 & EhRab7E protein \\
\hline Yes & 3.3411 & EHI_014030 & NAD(P) transhydrogenase subunit alpha, putative \\
\hline Yes & 2.6606 & EHI_025100 & G protein-coupled receptor 1 \\
\hline
\end{tabular}


Table 2. Cont.

\begin{tabular}{|c|c|c|c|}
\hline TM and/or Signal Peptide & Fold Change & Mapped ID & Gene Name \\
\hline Yes & 4.1994 & EHI_012270 & $170 \mathrm{kDa}$ surface lectin \\
\hline Yes & 2.3614 & EHI_141330 & Uncharacterized protein \\
\hline Yes & 2.5185 & EHI_120590 & Uncharacterized protein \\
\hline- & 2.0883 & EHI_041950 & Vacuolar protein sorting 35 , putative \\
\hline Yes & 2.8047 & EHI_164800 & Cysteine protease binding protein family 1 \\
\hline- & 3.411 & EHI_136160 & Calreticulin putative \\
\hline Yes & 3.1331 & EHI_098510 & Uncharacterized protein \\
\hline- & 4.4288 & EHI_192810 & small GTPase Rab7A \\
\hline- & 2.9434 & EHI_147010 & Long-chain-fatty-acid-CoA ligase, putative \\
\hline Yes & 2.3719 & EHI_071590 & Protein disulfide isomerase putative \\
\hline- & 2.2623 & EHI_133970 & Thioredoxin putative \\
\hline Yes & 2.8980 & EHI_158240 & 3-ketoacyl-CoA synthase \\
\hline Yes & 2.2738 & EHI_139030 & EhSyntaxin 1A (Fragment) \\
\hline- & 2.3774 & EHI_107250 & small GTPase Rab11B \\
\hline- & 3.3584 & EHI_140260 & Copine putative \\
\hline Yes & 2.2996 & EHI_054180 & Protein SEY1 homolog 2 \\
\hline Yes & 3.1250 & EHI_200230 & Cell surface protease gp63 putative \\
\hline- & 2.3851 & EHI_127030 & Uncharacterized protein \\
\hline- & 2.4601 & EHI_007330 & Beta-hexosaminidase \\
\hline Yes & 2.0039 & EHI_067090 & Uncharacterized protein \\
\hline Yes & 2.5342 & EHI_178470 & Cysteine protease binding protein family 6 \\
\hline Yes & 3.1223 & EHI_042370 & Galactose-specific adhesin $170 \mathrm{kDa}$ subunit, putative \\
\hline Yes & 2.7603 & EHI_021410 & EhSyntaxin B \\
\hline Yes & 3.8750 & EHI_133900 & Galactose-inhibitable lectin $170 \mathrm{kDa}$ subunit putative \\
\hline Yes & 3.4012 & EHI_148790 & Gal galNac lectin light subunit \\
\hline- & 2.7452 & EHI_175460 & $60 S$ acidic ribosomal protein $\mathrm{P} 0$ \\
\hline Yes & 2.4905 & EHI_101230 & P-glycoprotein 6 \\
\hline- & 2.5152 & EH_052400 & Elongation factor-1-alpha \\
\hline
\end{tabular}

\subsection{Functional Classification of Differential Abundant Proteins.}

Figure 1 shows $48.07 \%, 33.04 \%$ and $18.88 \%$ of the differential abundant proteins involved in cellular components, biological process and molecular function, respectively. Increased abundance proteins of the membrane fraction involved in molecular function included catalytic activity (seven proteins), binding (seven proteins), structural molecular activity (one protein) and transporter activity (one protein) (Figure 2A). Figure 2B showed increased abundance proteins involved in the biological processes such as biological regulation (four proteins), biogenesis (six proteins), cellular process (eleven proteins), developmental process (one protein), localization (four proteins), metabolic process (five proteins), response to stimulus (three proteins), and signaling (three proteins). These proteins were also involved in cellular components such as cell part (twelve proteins), cell (twelve proteins), membrane part (five proteins), membrane (nine proteins), membraneenclosed lumen (one protein), organelle part (three proteins), organelle (ten proteins), and protein-containing complex (six proteins), as shown in Figure 2C. 
Table 3. Decreased abundance of cytosolic proteins in the membrane versus cytosolic fractions $(\mathrm{q}<0.05)$. These proteins lack a transmembrane (TM) and signal peptide domain.

\begin{tabular}{|c|c|c|c|}
\hline Mapped ID & Fold Change & Gene Name/Symbol & $\begin{array}{c}\text { PANTHER } \\
\text { (Family/Subfamily) }\end{array}$ \\
\hline EHI_130700 & 2.953 & Enolase putative & Enolase (Pthr11902:Sf1) \\
\hline EHI_052860 & 2.9703 & Heat shock protein 70 putative & $\begin{array}{l}\text { Ribosome-Associated Molecular Chaperone Ssb1-Related } \\
\text { (Pthr19375:Sf395) }\end{array}$ \\
\hline EHI_104390 & 2.6725 & Actin binding protein putative & - \\
\hline EHI_042170 & 3.2691 & Aminoacyl-histidine dipeptidase putative & Cytosol Non-Specific Dipeptidase (Pthr43501:Sf1) \\
\hline EHI_048310 & 2.4029 & EhSec24C & Secretory 24cd, Isoform C (Pthr13803:Sf38) \\
\hline EHI_023110 & 3.1633 & NADP-dependent Alcohol dehydrogenase & Dehydrogenase 1, Putative-Related (Pthr42813:Sf4) \\
\hline EHI_125840 & 3.0822 & Peptidyl-prolyl cis-trans isomerase & $\begin{array}{l}\text { Peptidyl-Prolyl Cis-Trans Isomerase D-Related } \\
\text { (Pthr11071:Sf380) }\end{array}$ \\
\hline EHI_166810 & $3.7550 / 2.6014$ & Elongation factor 2 & Elongation Factor 2 (Pthr42908:Sf10) \\
\hline EHI_098570 & 3.1517 & Fructose 16 -bisphosphate aldolase putative & $\begin{array}{c}\text { D-Tagatose-1,6-Bisphosphate Aldolase Subunit Gaty-Related } \\
\text { (Pthr30304:Sf0) }\end{array}$ \\
\hline EHI_155410 & 2.8691 & $40 \mathrm{~S}$ ribosomal protein S11, putative & 40s Ribosomal Protein S11 (Pthr10744:Sf9) \\
\hline EHI_104630 & 2.5185 & Filamin 2 putative & Zgc:100997 (Pthr19961:Sf58) \\
\hline EHI_006670 & 2.5009 & Uncharacterized protein & Expressed Protein (Pthr19308:Sf14) \\
\hline EHI_198930 & 3.7526 & Actin putative & Actin-Related Protein 3b (Pthr11937:Sf31) \\
\hline EHI_021360 & 2.5234 & Uncharacterized protein & Translationally Controlled Tumor Protein (Pthr11991:Sf0) \\
\hline EHI_192450 & 2.0262 & Rho family GTPase & Ras-Related Protein Rac1-Related (Pthr24072:Sf281) \\
\hline EHI_146180 & 2.3813 & Rho family GTPase & Ras-Related Protein Rac1-Related (Pthr24072:Sf281) \\
\hline EHI_104560 & 2.8300 & Cortexillin putative & Cortexillin-2 (Pthr23167:Sf67) \\
\hline EHI_176140 & $2.7438 / 2.4697$ & Profilin & Profilin (Pthr11604:Sf0) \\
\hline EHI_174180 & 2.1882 & Skp1 family protein & S-Phase Kinase-Associated Protein 1 (Pthr11165:Sf24) \\
\hline EHI_165350 & 2.6236 & Malate dehydrogenase putative & $\begin{array}{l}\text { Hydroxycarboxylate Dehydrogenase B-Related } \\
\text { (Pthr11091:Sf0) }\end{array}$ \\
\hline EHI_159160 & 2.4921 & Superoxide dismutase & Superoxide Dismutase [Fe] 2, Chloroplastic (Pthr42769:Sf3) \\
\hline EHI_011270 & 2.2522 & Uncharacterized protein & Si:Ch211-282j17.12-Related (Pthr18884:Sf83) \\
\hline EHI_178960 & $2.7608 / 2.5979$ & Acetyl-CoA synthetase, putative & Acetate-Coa Ligase [Adp-Forming] (Pthr43334:Sf1) \\
\hline EHI_152680 & 2.1693 & EH-domain containing protein putative & At21416p (Pthr11216:Sf31) \\
\hline EHI_147570 & $2.1748 / 2.7106$ & Rho GDP exchange inhibitor, putative & Ld16419p (Pthr10980:Sf3) \\
\hline EHI_193330 & 2.2123 & $60 \mathrm{~S}$ ribosomal protein $\mathrm{L} 23$ putative & 60s Ribosomal Protein L23 (Pthr11761:Sf8) \\
\hline EHI_110180 & 2.4310 & Myosin heavy chain & Myosin Heavy Chain, Non-Muscle (Pthr45615:Sf40) \\
\hline EHI_051060 & 3.7812 & Pyruvate ferredoxin oxidoreductase & Pyruvate-Flavodoxin Oxidoreductase-Related (Pthr32154:Sf0) \\
\hline EHI_125950 & 3.1278 & Alcohol dehydrogenase putative & Alcohol Dehydrogenase Yqhd (Pthr43633:Sf1) \\
\hline EHI_006810 & 2.7083 & 14-3-3 protein 3 & 14-3-3 Protein Zeta (Pthr18860:Sf106) \\
\hline EHI_152650 & 2.5095 & Type A flavoprotein, putative & Diflavin Flavoprotein A 2-Related (Pthr32145:Sf11) \\
\hline EHI_044970 & 3.9670 & Malic enzyme & Nadp-Dependent Malic Enzyme (Pthr43237:Sf4) \\
\hline EHI_091250 & 2.8148 & Arp2/3 complex 34 kDa subunit & Actin-Related Protein 2/3 Complex Subunit 2 (Pthr12058:Sf0) \\
\hline EHI_005020 & 2.5429 & F-actin-capping protein subunit beta & F-Actin-Capping Protein Subunit Beta (Pthr10619:Sf0) \\
\hline EHI_009530 & 2.136 & Pyruvate, phosphate dikinase & $\begin{array}{l}\text { Pyruvate, Phosphate Dikinase 1, Chloroplastic } \\
\text { (Pthr22931:Sf9) }\end{array}$ \\
\hline
\end{tabular}




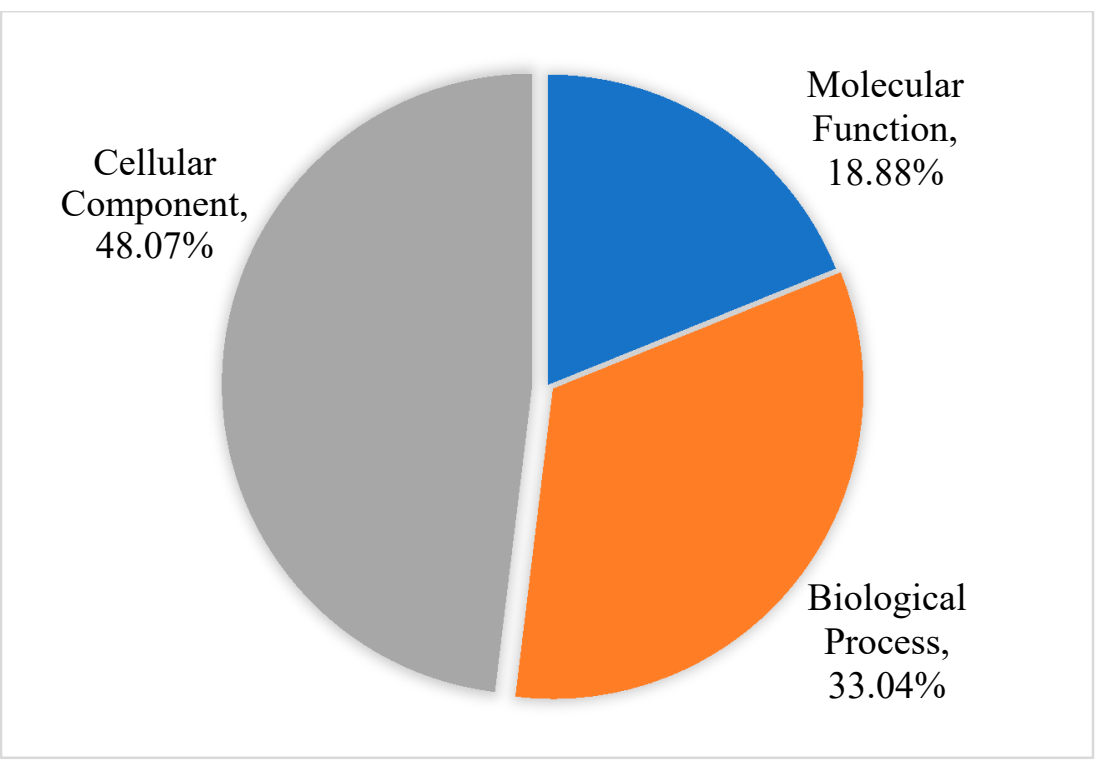

Figure 1. The functional analysis of increased and decreased abundance proteins in the membrane versus cytosolic fractions.

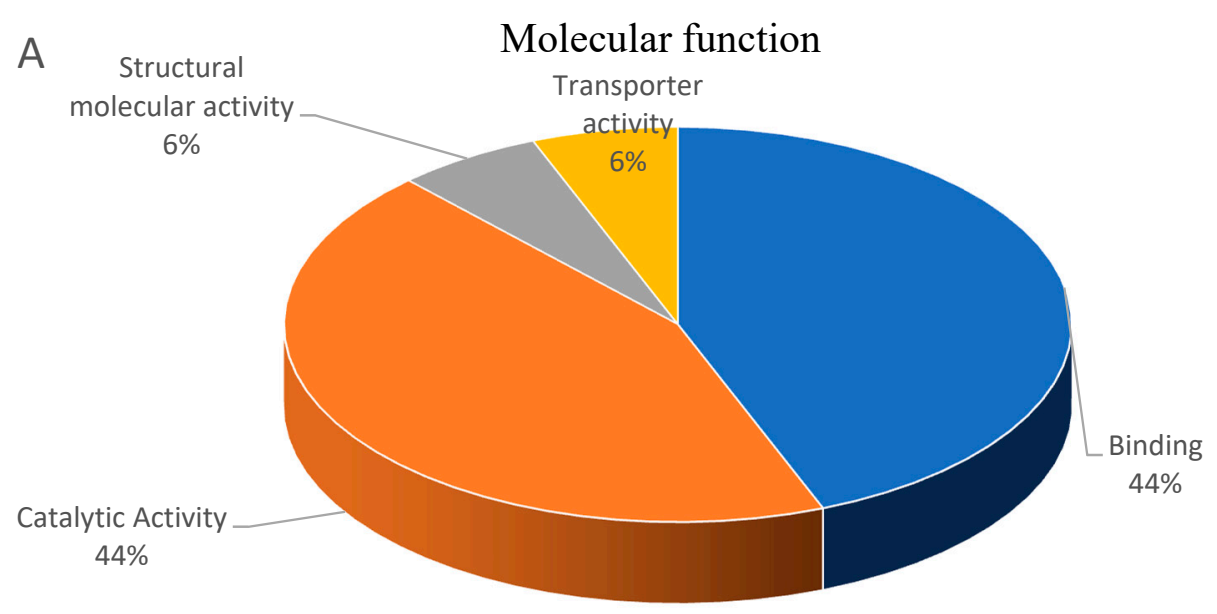

B

\section{Biological process}

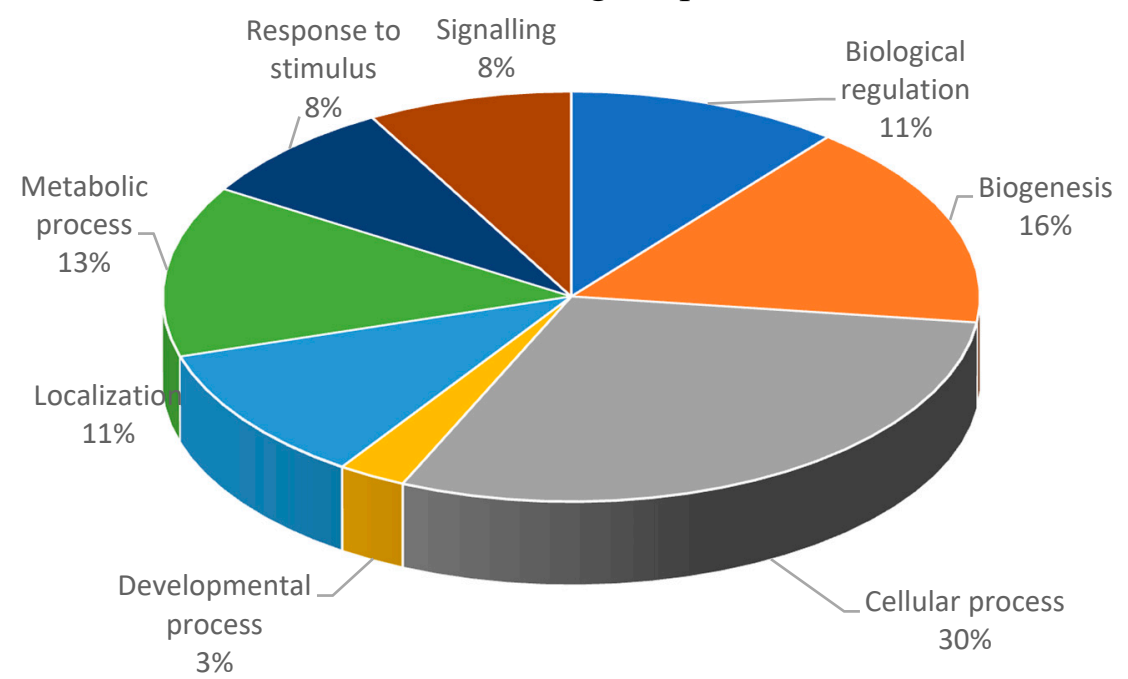

Figure 2. Cont. 


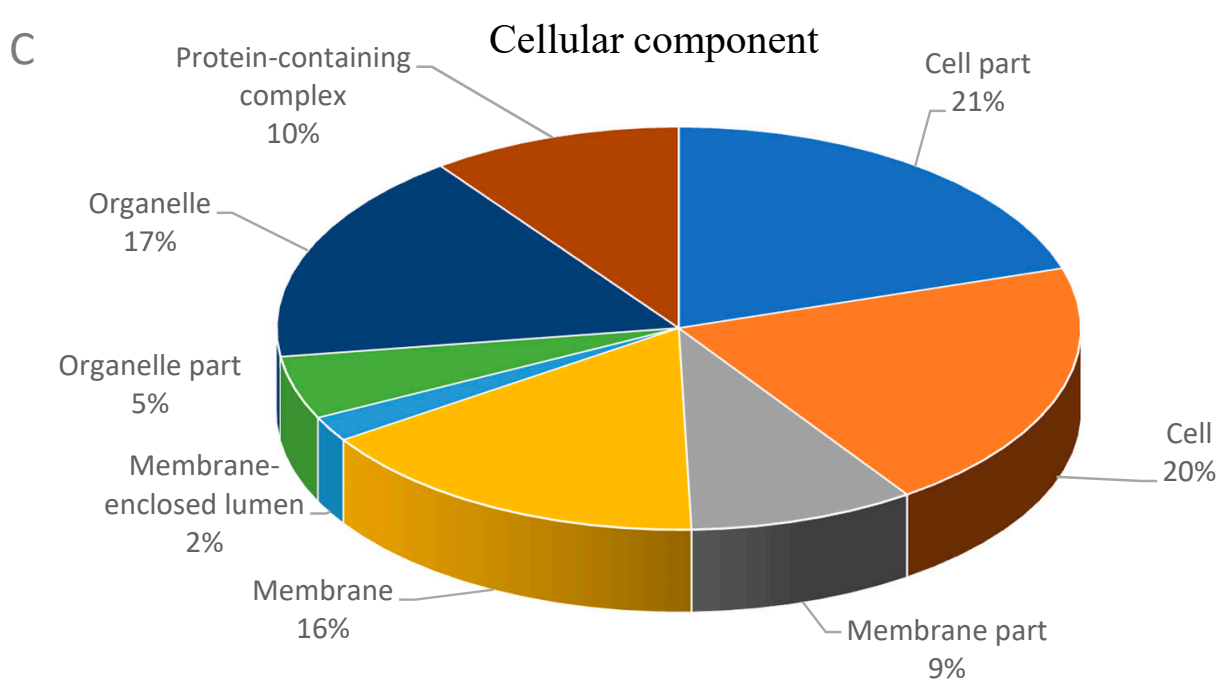

Figure 2. Functional classification analysis of increased abundance proteins according to their molecular function (A), biological process (B), and cellular component (C).

Decreased abundance proteins were involved in the molecular function such as binding (fourteen proteins), catalytic activity (nine proteins), molecular function regulator (one protein), structural molecular activity (three proteins), and translation regulator activity (one protein) (Figure 3A). Proteins associated with biological process involved biological regulation (seven proteins), biogenesis (seven proteins), cellular process (twelve proteins), developmental process (three proteins), localization (one protein), metabolic process (two proteins), response to stimulus (five proteins), and signaling (three proteins) (Figure 3B). These proteins were classified into cellular components such as cell and cell part (fifteen proteins each), membrane (two proteins), organelle and organelle part (nine and six proteins each), protein-containing complex (six proteins), and supramolecule complex (one protein) as shown in Figure 3C.

Molecular function

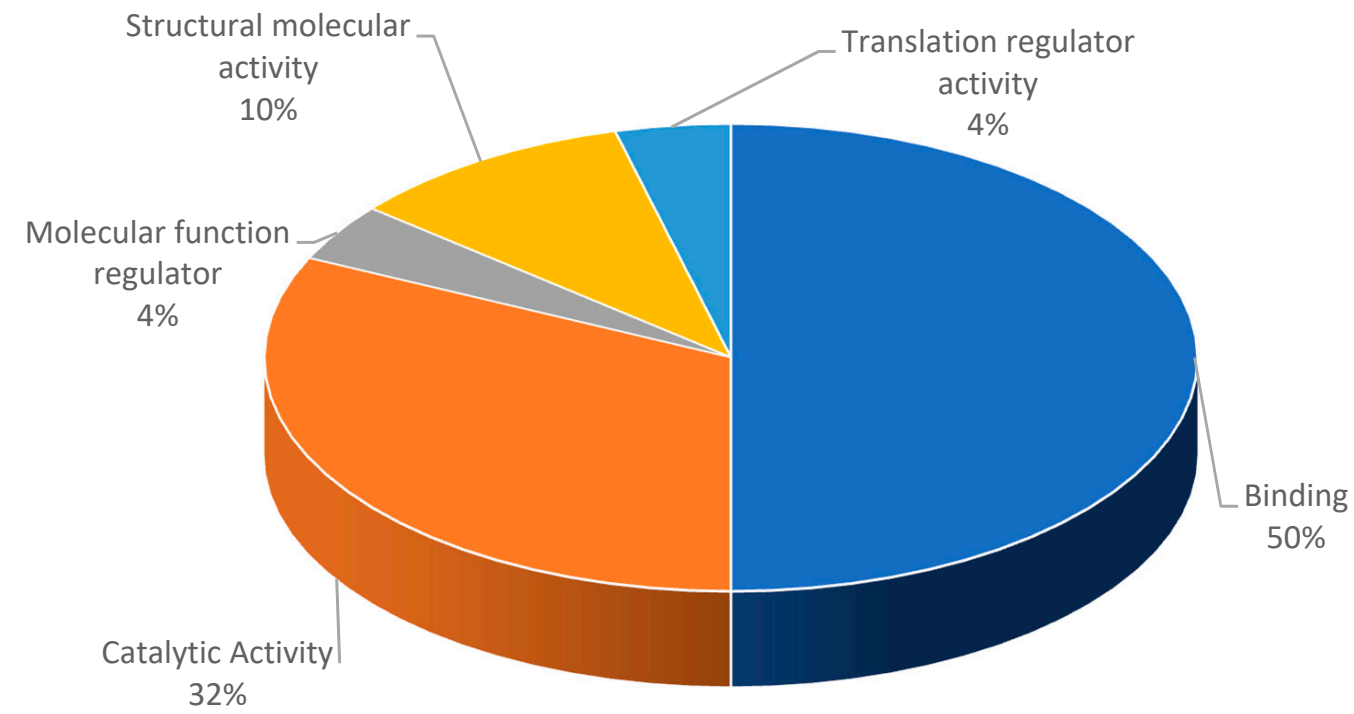

Figure 3. Cont. 
B Biological process

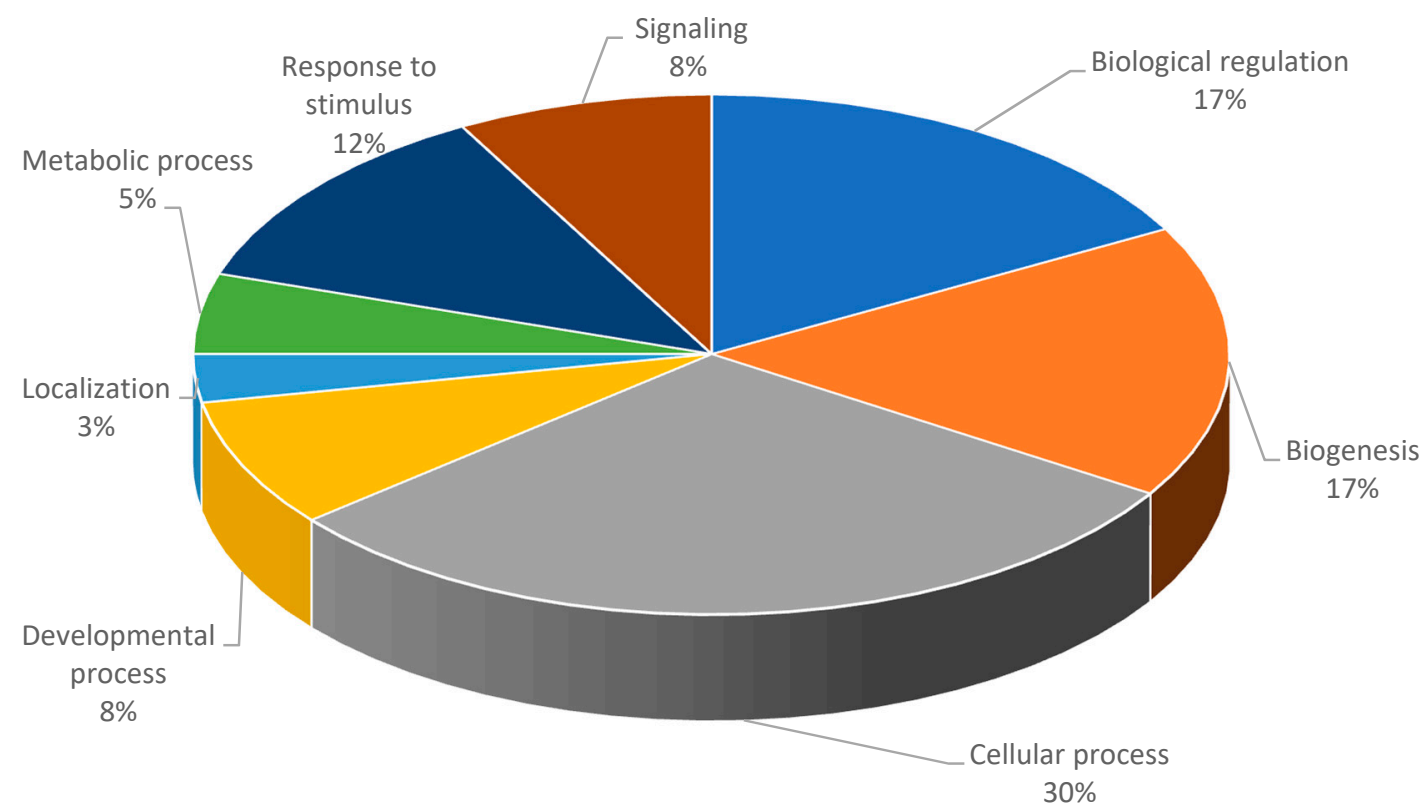

C

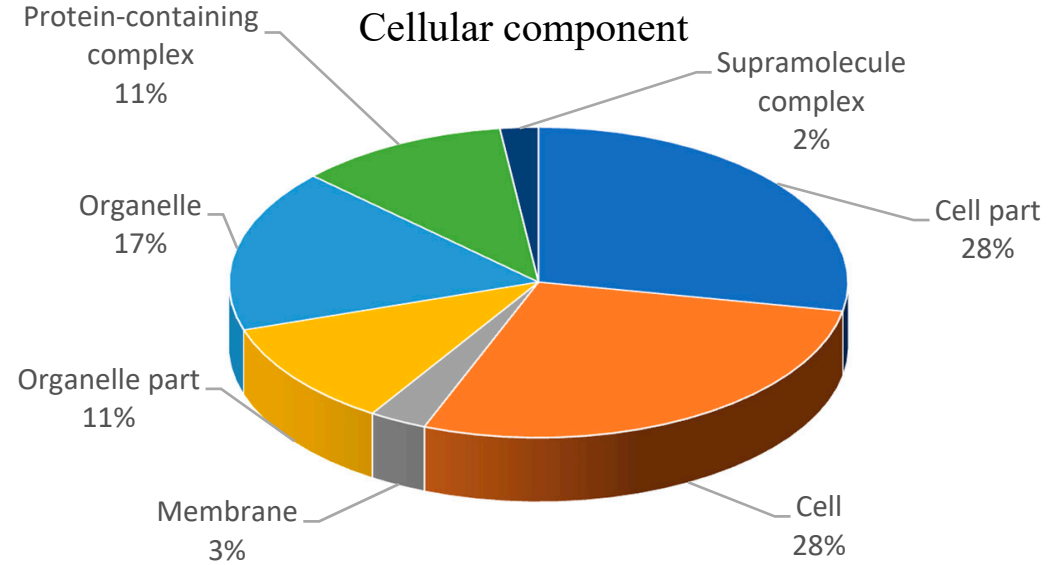

Figure 3. Functional classification of decreased abundance proteins according to their molecular function (A), biological process (B) and cellular component (C).

Increased abundance proteins demonstrated by PANTHER DB analysis (Table S1 in Supplementary Materials) were mainly involved in binding and catalytic activity (molecular function), cellular process (biological process) and cell and cell part (cellular component). The identified proteins were Rho family GTPase protein (EHI_129750), $70 \mathrm{kDa}$ heat shock protein, putative (EHI_199590), calreticulin putative (EHI_136160), 60S acidic ribosomal protein (EHI_175460), NAD(P) transhydrogenase subunit alpha putative (EHI_014030), 3-oxo 5 alpha-steroid 4-dehydrogenase domain (EHI_076870), calcium- transporting ATPase (EHI_054830), Ehsyntaxin 1A fragment (EHI_139030) and protein SEY1 homolog 2 (EHI_054180).

On the other hand, PANTHER DB analysis showed decreased abundant proteins (Table S2 in Supplementary Materials) mainly involved in binding (molecular function), cellular process (biological process) and cell and cell part (cellular component). There were some proteins involved in the binding, cellular activities and cell/cell part such as heat shock protein 70 putative (EHI_052860), peptidyl-prolyl cis-trans isomerase (EHI_125840), 
elongation factor 2 (EHI_166820), filamin 2 putative (EHI_104630), actin putative (EHI_198930), Rho family GTPase (EHI_192450 and EHI_146180), profilin (EHI_176140), Skp1 family protein (EHI_174180), Rho GDP exchange inhibitor (EHI_147570), Arp2/3 complex 34 kDa subunit (EHI_091250) and F-actin-capping protein subunit beta (EHI_005020).

\subsection{Protein-Protein Interaction Network Prediction.}

Highly significant interactions $\left(p<3.66 \times 10^{-11}\right)$ were predicted in decreased abundance proteins when compared to increased abundance proteins $\left(p<1.91 \times 10^{-7}\right)$ (Figure 4A,B). Analysis by STRiNG showed there were interactions between membrane and cytosolic proteins among increased abundance proteins as shown in Figure 4A. Line colors indicate types of interaction evidences (Table 4 ).

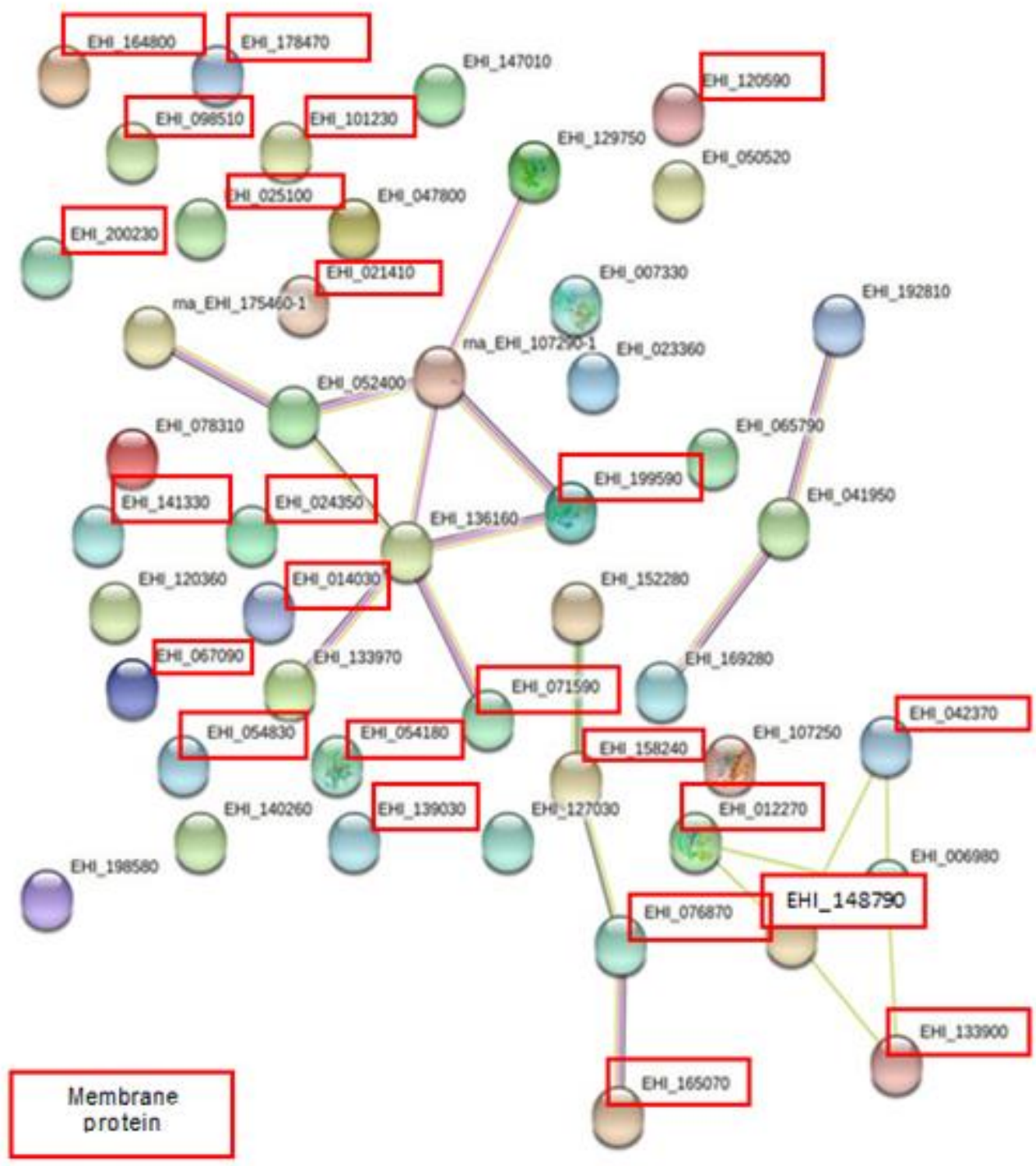

(A)

Figure 4. Cont. 


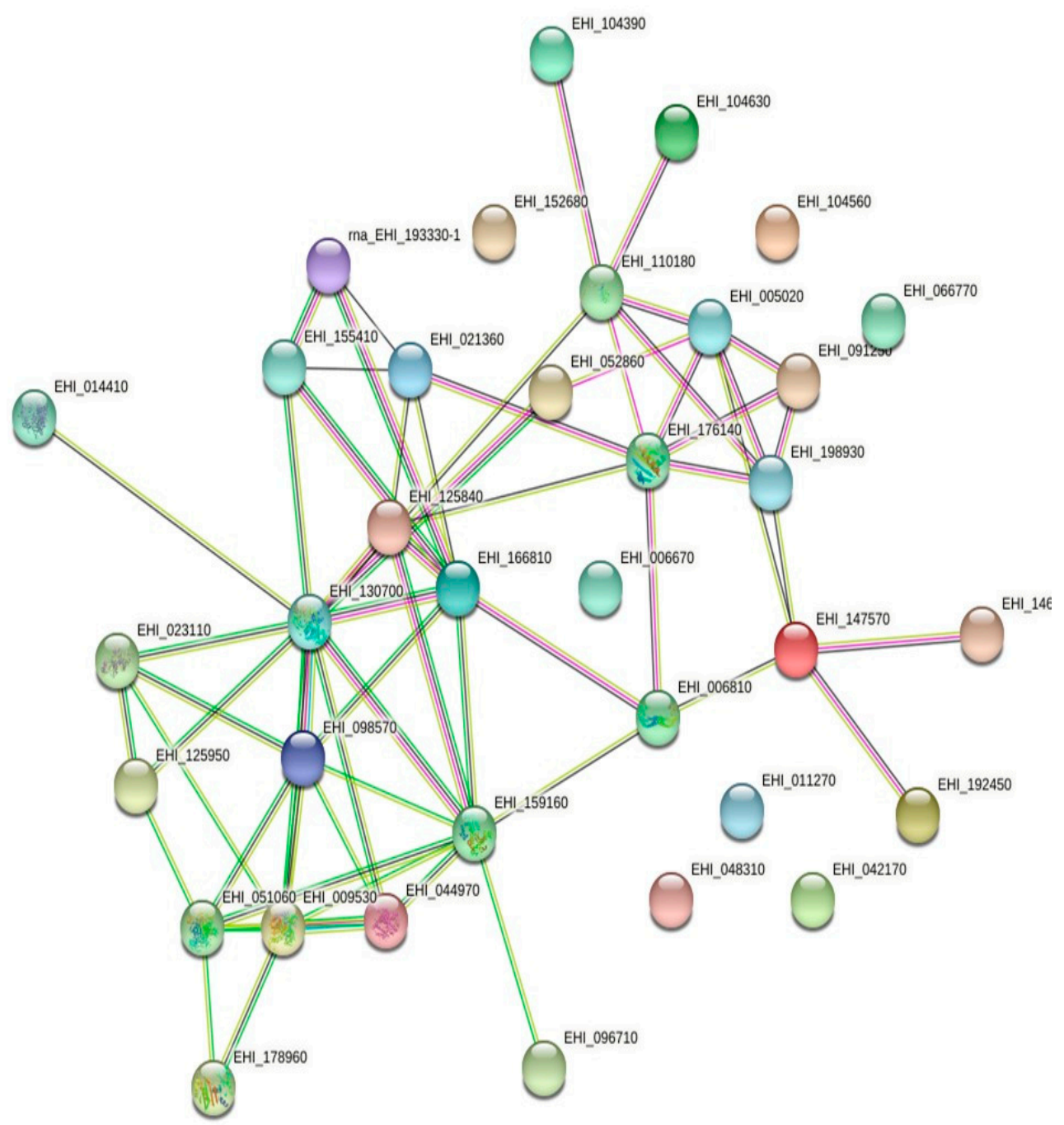

(B)

Figure 4. STRiNG generated networks of increased and decreased abundance proteins. (A) Increased abundance proteins comprised membrane and cytosolic proteins. Proteins with accession no. in the red boxes are membrane proteins. Meanwhile, proteins with accession no. without red boxes are cytosolic proteins. (B) Decreased abundance proteins comprised cytosolic proteins.

Table 4. Line colors explanation.

\begin{tabular}{ccc}
\hline Color & Evidence \\
\hline Red & Presence of fusion \\
Green & Neighborhood \\
Blue & Cooccurrence \\
Purple & Experimental \\
Yellow & Text mining \\
Light blue & Database \\
Black & Coexpression \\
\hline
\end{tabular}

Note: Different color lines indicate types of interaction evidence used in predicting the association.

\section{Discussion}

In this study, there were increased and decreased abundance proteins in membrane versus cytosolic fractions from combining all three extraction methods. In this study, $24(44.4 \%)$ out of 54 increased abundance proteins in the membrane fraction were confirmed membrane proteins as they had a transmembrane region and/or single peptide 
(Tables 3 and 4). Meanwhile, 45 (100\%) of the decreased abundance proteins were cytosolic proteins without a transmembrane region and/or single peptide. This study supported our previous finding (Ujang et al., (2018) [7]) in which there were mixed membrane and cytosolic proteins in the membrane fraction. We have confirmed and added value to the previous report that several increased abundance cytosolic proteins are present in the $E$. histolytica membrane fraction in this study.

There was a combination of membrane and cytosolic proteins among increased abundance proteins. Membrane proteins are $70 \mathrm{kDa}$ HSP putative, 3-oxo 5-alpha-steroid 4dehydrogenase, calcium-transporting ATPase, NAD $(\mathrm{P})$ transhydrogenase, protein SEY1 homolog 2, cell surface proteases gp63 putative, Ehsyntaxin 1A (fragment) and Ehsyntaxin B. Furthermore, Rho family GTPase, calreticulin putative, $60 \mathrm{~S}$ acidic ribosomal protein, uncharacterized proteins, and Rho and Rab family GTPase were cytosolic proteins (Table 2). For example, a well-studied protein that related to the parasite's pathogenicity over past decades was calreticulin and this protein was found to be increased in abundance in the present study. Calreticulin is an immunogenic molecule involved in binding and cellular process, has no transmembrane domain and/or signal peptide. It is able to induce a host immune response. Furthermore, during the initial stage of the infection, increased expression of this parasite protein can be seen $[8,9]$.

In this study, type A flavoprotein (EHI_152650), an oxidoreductase enzyme, was found to be decreased in abundance together with other stress response enzymes; superoxide dismutase (EHI_159160), peptidylprolyl isomerase (EHI_125840), and heat shock protein 70 putative (EHI_052860) in the membrane fraction. An interesting observation by Macfarlane and Singh (2005) showed the stress response in the nonvirulent $E$. histolytica was affected if a group of genes decreased in their expression, for instance, type A flavoprotein, which plays a role in the detoxification of oxygen and nitric oxide [8].

Different isoforms of Rho family GTPase and $70 \mathrm{kDa}$ heat shock proteins were found to be increased and decreased in abundance in this study (Tables 2 and 3). The Rho family GTPase protein (EHI_129750) and $70 \mathrm{kDa}$ heat shock protein putative (EHI_199590) were increased in abundance, while its isoforms Rho family GTPase (EHI_192450 and EHI_146180) and heat shock protein 70 putative (EHI_052860) were decreased in abundance by comparing the two fractions. The $70 \mathrm{kDa}$ heat shock protein and Rho family GTPase family proteins play important roles in the virulence of the parasite together with other identified proteins, for example, Gal/GalNAc subunit, NAD(P) transhydrogenase alpha and calreticulin [9]. From the functional classification analysis, Rho family GTPase (EHI_129750) is involved in catalytic activity and binding and increased abundance of GTPase proteins may give a possibility of effective colonization and invasion of the trophozoites in the host [10]. Soid-Raggi et al., (1998) reported that the G protein that the Rho family GTPase belongs has the possible elements of signal transduction in the trophozoite's interaction with fibronectin [10]. Meanwhile, increased abundance of $70 \mathrm{kDa}$ heat shock protein will protect amoeba because the heat shock protein (HSP) will be produced if an immediate temperature spike occurs [11].

Amoeba also contains peroxiredoxin (Prx), superoxide dismutase (EHI_159160), flavoprotein A (EHI_096710), ferredoxin (EHI_051060), thioredoxin (EHI_133970) and thioredoxin reductase for its protection against oxidative stress. Furthermore, the pathogenesis of Entamoeba has a strong association with antioxidative defense mechanisms. In this study, only the thioredoxin enzyme, which is known as a sensitive buffer was found to be increased in abundance [12]. Meanwhile, superoxide dismutase, flavoprotein A and ferredoxin were found to be decreased in abundance. Superoxide dismutase plays an important role in demolishing the cell radical of superoxide anions, which harms the $E$. histolytica biological system.

Another cytosolic protein, F-actin-capping protein subunit beta (EHI_005020), is a significant protein that has been detected because it takes roles in many functions including binding activities (molecular function); cellular process, biogenesis, and biological regu- 
lation (biological process); and cell/cell part (cellular component), which can inhibit the elongation of actin filaments to assure the parasite is motile [13].

Highly significant interactions $\left(p<3.66 \mathrm{e} \times 10^{-11}\right)$ were predicted in decreased abundance proteins when compared to increased abundance proteins $\left(p<1.91 \times 10^{-7}\right)$ (Figure 4A,B). This result is because all of the decreased abundance proteins are cytosolic proteins, which have been extensively studied previously. The results showed a few membrane and cytosolic proteins in the membrane fraction were predicted to be associated by the protein-protein interaction analysis.

Interesting interactions included calreticulin (EHI_136160) as a central protein, which was linked to two membrane proteins and three cytosolic proteins (Figure 4A). Calreticulin, a cytosolic protein, interacted with two membrane proteins, 70 kDa HSP (EHI_199590) and protein disulfide isomerase (EHI_071590) (Figure 4A). EhPDI (protein disulfide isomerase) belongs to the PDI family, which is known as ER's lumen soluble marker. There was a report by Salgado et al., (2005) where a nuclear fraction of the amoeba was associated with the lack of endoplasmic reticulum (ER) markers. Calreticulin is an ER protein specifically located at the membrane of the ER periphery [14] and it promotes sites for glycosylation and calcium-binding. Overexpression of this protein in patients with amoebic liver abscess was associated with an immunogenic response at the initial stage of infection [15]. Both PDI and calreticulin associated with ER, which explains the possibility of the interaction occuring between these proteins in this study. On the other hand, calreticulin linked to other proteins such as $70 \mathrm{kDa} H S P$, actin, thioredoxin and elongation factor 1 due to these proteins being involved in similar molecular functions and biological processes categories. For instance, both $70 \mathrm{kDa}$ HSP and calreticulin were involved in binding and cellular activity.

\section{Conclusions}

In conclusion, this study has confirmed the presence of mixed differential abundance proteins in membrane versus cytosolic fractions. Furthermore, the functional analysis and protein-protein interactions among these proteins were predicted. Several membrane and cytosolic proteins that showed significant interactions merit further investigation to confirm their localization and function on the membrane part to further our understanding of the E. histolytica pathogenesis.

Supplementary Materials: The following are available online at https:/ /www.mdpi.com/article/ 10.3390/membranes11060376/s1, Supplementary file 1. Table S1. Classification of Increased Abundance Proteins by PANTHER DB. Table S2. Classification of decreased abundance proteins by PANTHER DB.

Author Contributions: Conceptualization, N.O.; methodology, N.A. and N.O.; software, N.A.; validation, N.O. and N.A.; formal analysis, N.A.; investigation, N.A.; resources, N.O.; data curation, N.A.; writing —original draft preparation, N.A.; writing—review and editing, N.O.; visualization, N.A.; supervision, N.O.; project administration, N.O.; funding acquisition, N.O. All authors have read and agreed to the published version of the manuscript.

Funding: This research was funded by Malaysian Ministry of Higher Education Fundamental Research Grant (FRGS), grant number 203.CIPPM.6711835 and Universiti Sains Malaysia.

Institutional Review Board Statement: Not applicable.

Informed Consent Statement: Not applicable.

Data Availability Statement: Not applicable.

Acknowledgments: We would like to acknowledge Malaysian Ministry of Health for providing a fellowship to Norhidayah Azmi during her study.

Conflicts of Interest: The authors declare no conflict of interest. 


\section{References}

1. Biller, L.; Matthiesen, J.; Kühne, V.; Lotter, H.; Handal, G.; Nozaki, T.; Saito-Nakano, Y.; Schümann, M.; Roeder, T.; Tannich, E.; et al. The Cell Surface Proteome of Entamoeba histolytica. Mol. Cell. Proteom. 2014, 13, 132-144. [CrossRef] [PubMed]

2. Laura, M.; Upinder, S. Entamoeba histolytica: A snapshot of current research and methods for genetic analysis. Curr. Opin. Microbiol. 2012, 15, 469-475.

3. Ibne Karim, M.A.; Rashidul, H.; Abdullah, S.; Mamun, K.; Nicholas, E.S.; Sean, A.G.; Gerard, A.C.; William, A.P., Jr. Proteomic analysis of the cyst stage of Entamoeba histolytica. PLoS Negl. Trop. Dis. 2012, 6, e1643.

4. Krogh, A.; Larsson, B.; von Heijne, G.; Sonnhammer, E.L. Predicting transmembrane protein topology with a hidden markov model: Application to complete genomes. J. Mol. Biol. 2001, 305, 567-580. [CrossRef] [PubMed]

5. Tsirigos, K. Bioinformatics Methods for Topology Prediction of Membrane Proteins. Ph.D. Thesis, Department of Biochemistry and Biophysics, Stockholm University, Stockholm, Sweden, 2017.

6. Zhang, Q.C.; Petrey, D.; Garzón, J.I.; Deng, L.; Honig, B. PrePPI: A structure-informed database of protein-protein interactions. Nucleic Acids Res. 2012, 41, D828-D833. [CrossRef] [PubMed]

7. Ujang, J.; Sani, A.A.A.; Lim, B.H.; Noordin, R.; Othman, N. Analysis of Entamoeba histolytica Membrane Proteome Using Three Extraction Methods. Proteomics 2018, 18, e1700397. [CrossRef] [PubMed]

8. Macfarlane, R.C.; Singh, U. Identification of Differentially Expressed Genes in Virulent and Nonvirulent Entamoeba Species: Potential Implications for Amebic Pathogenesis. Infect. Immun. 2006, 74, 340-351. [CrossRef] [PubMed]

9. Ng, Y.L.; Olivos-García, A.; Lim, T.K.; Noordin, R.; Lin, Q.; Othman, N. Entamoeba histolytica: Quantitative Proteomics Analysis Reveals Putative Virulence-Associated Differentially Abundant Membrane Proteins. Am. J. Trop. Med. Hyg. 2018, 99, 1518-1529. [CrossRef] [PubMed]

10. Soid-Raggi, L.G.; Torres-Márquez, M.E.; Meza, I. Entamoeba histolytica: Identification of functional G(s) and G(i) proteins as possible signal transduction elements in the interaction of trophozoites with fibronectin. Exp. Parasitol. 1998, 90, 262-269. [CrossRef] [PubMed]

11. Ximénez, C.; González, E.; Nieves, M.; Magaña, U.; Morán, P.; Gudiño-Zayas, M. Differential expression of pathogenic genes of E. histolytica versus E. dispar in a model of infection using human liver tissue explants. PLoS ONE 2017, 12, e0181962.

12. Schlosser, S.; Leitschm, D.; Duchêne, M. Entamoeba histolytica: Identification of thioredoxin-targeted proteins and analysis of serine acetyltransferase-1 as a prototype example. Biochem. J. 2013, 451, 277-288. [CrossRef] [PubMed]

13. Isenberg, G.; Aebi, U.; Pollard, T.D. An action-binding protein from Acanthamoeba regulates actin filament polymerization and interactions. Nature 1980, 288, 455-459. [CrossRef] [PubMed]

14. Salgado, M.; Villagómez-Castro, J.C.; Rocha-Rodríguez, R.; Sabanero-Lopez, M.; Ramos, M.A.; Alagón, A.; López-Romero, E.; Sánchez-López, R. Entamoeba histolytica: Biochemical and molecular insights into the activities within microsomal fractions. Exp. Parasitol. 2005, 110, 363-373. [CrossRef] [PubMed]

15. Gonzalez, E.; Rico, G.; Mendoza, G.; Ramos, F.; Garcıa, G.; Moran, P.; Valadez, A.; Melendro, E.I.; Ximenez, C. Calreticu-lin-like molecule in trophozoites of Entamoeba histolytica HM1: IMSS (Swissprot: Accession P83003). Am. J. Trop. Med. Hyg. 2002, 67, 636-639. [CrossRef] [PubMed] 